\title{
ORGANIZACIÓN ADMINISTRATIVA Y PRODUCTIVIDAD DE LAS LIGAS DEPORTIVAS BOGOTANAS
}

\section{ADMINISTRATIVE ORGANIZATION AND PRODUCTIVITY OF BOGOTA'S SPORTS LEAGUES}

\author{
José Ramos Acosta ${ }^{1}$ \\ ${ }^{1}$ Licenciado en Educación Física, Especialista en administración deportiva y en Docencia Universitaria, Magister en Ciencias \\ del Deporte Docente e investigador de planta. Universidad de Ciencias Aplicadas y Ambientales U.D.C.A, e-mail: joramos@ \\ udca.edu.co; (iD https://orcid.org/0000-0003-2693-8315
}

\author{
Rev. U.D.C. Act. \& Div. Cient. 21(2):319-327, Julio-Diciembre, 2018 \\ https://doi.org/10.31910/rudca.v21.n2.2018.1081
}

Artículo de acceso abierto publicado por Revista U.D.C.A Actualidad \& Divulgación Científica

bajo una licencia Creative Commons CC BY-NC 4.0

\section{RESUMEN}

La labor fundamental de las Ligas deportivas es reunir los deportistas que llegan de los diferentes clubes afiliados y posibilitarles actividades de competencia continua, para detectar su talento deportivo y seleccionar, a partir de esta caracterización, a los de mayor nivel técnico, para conformar los seleccionados de la ciudad, en los distintos deportes y presentarlos a las competencias departamentales y nacionales, donde serán nuevamente escogidos, para la alta competencia, a nivel de federaciones. El presente trabajo tuvo como objetivo fundamental reconocer la desigualdad existente en las ligas deportivas bogotanas, en cuanto a desarrollo organizacional, administrativo y de productividad, lo cual, no permite que el deporte en la ciudad evolucione, de manera equilibrada, siendo importante encontrar la raíz de esta problemática, toda vez que la organización deportiva de las ligas debe ser eficiente y de alta productividad, para que el país deportivo tenga un mayor número de atletas de alto rendimiento, que lo represente en justas internacionales, mundiales y olímpicas, con buenos resultados. Con este estudio realizado, con un enfoque metodológico mixto, se logró registrar que la productividad deportiva en las Ligas de Bogotá no es uniforme y tampoco su desarrollo administrativo, lo que, seguramente, no ha permitido una evolución equilibrada del deporte capitalino.

Palabras clave: ligas deportivas, organización administrativa, desarrollo organizacional, productividad.

\section{ABSTRACT}

The fundamental task of the sports leagues is to gather the athletes that come from the different affiliated clubs and enable them to continue competition activities, to detect their sporting talent and select from this detection the highest technical level, to conform the selected ones of the city in the different sports and present them to the departmental and National level competitions, where they will be selected again for the high competition. The main objective of this work was to recognize the inequality that exists in Bogotá's sports leagues, in terms of organizational, administrative and productivity development, which does not allow sport in the city to evolve in a balanced manner, It is important to find the root of this problem, since the sports organization of the leagues must be efficient and highly productive so that the sporting country has a greater number of high performance athletes that represent it in international, world and Olympic jousts. With this study carried out with a mixed methodological approach, it was possible to register that the sports productivity in the Leagues in the city of Bogotá is not uniform and neither its administrative development, which surely has not allowed a balanced evolution of the capital sport.

Keywords: sports leagues, organizational development, productivity.

\section{INTRODUCCIÓN}

De acuerdo con los dictámenes de la constitución colombiana, el derecho a la realización de actividad deportiva debe ser para todos los colombianos sin ningún tipo de discriminación y le corresponde al Estado desarrollar procesos, 
que faciliten y promuevan, entre la población, este tipo de actividades, en procura de una calidad de vida equilibrada y de mejor desarrollo para el ser humano (Constitución Política de Colombia, 1991). En concordancia con la constitución, aparece en el país el marco general de la ley 181 de 1995 que, de manera concreta, crea un espectro de posibilidades para el desarrollo amplio y armónico de las actividades de índole deportivo, donde aparecen, fundamentalmente, las Ligas Deportivas, como parte esencial del deporte asociado, mostrando el diseño institucional, constituido a nivel nacional, para que un deportista pueda acceder a la representación del país.

De acuerdo con lo anterior, las ligas deportivas son esenciales en el diseño del deporte asociado y tienen como objetivo el desarrollo de un deporte específico; sin embargo, según un estudio de Mesa et al. (2010), solo el 48\% de las ligas privilegia el deporte competitivo, pues, por lo general, lo hacen por desarrollo comunitario e, incluso, por recreación.

Ya Gutiérrez (2007), después de realizar un estudio investigativo en el área del deporte, afirmó que la organización administrativa, de cualquier ente deportivo, es esencial para su desarrollo general, así como es importante el conocimiento adquirido por sus directivos con relación a la administración y sus fundamentos, para que se logren alcanzar las metas plasmadas en la misión y la visión de las instituciones. Es necesaria, además, la aplicación del ciclo administrativo, desarrollando éstos criterios, con base en el conocimiento profundo de las ciencias administrativas, entendidas como un conjunto de actividades sociales, dirigidas a aprovechar los recursos existentes, de manera eficiente, con el propósito de alcanzar los objetivos perseguidos, con el mínimo de esfuerzo (Martínez Crespo, 2005).

Los procesos administrativos del deporte deben tener, como base, elementos esenciales de planeación, de organización y de dirección, procurando alcanzar la productividad, que es fundamental en este estudio, que se confunde, con frecuencia, con el término producción. Muchas personas piensan que a mayor producción más productividad, pero no es necesariamente cierto. Producción, se refiere a la actividad de producir bienes y servicios y productividad hace referencia a la utilización eficiente de los recursos al producir bienes o servicios (Omana del Castillo, 2003). Para autores destacados en el mundo de la administración, como Koontz et al. (2012), la productividad hace parte fundamental del control y, muchas compañías exitosas, crean su valor agregado, mediante la vigilancia cuidadosa de las operaciones productivas.

Este estudio se originó en Bogotá, D.C., en el 2017, donde se reconocieron las diferencias existentes en cuanto a productividad deportiva en las Ligas y se relacionó esta variable con la organización administrativa que se desarrolla en ellas, analizando, además, el desarrollo organizacional sobresaliente, que habían logrado algunas de ellas. De esta manera, al reconocer la relación existente entre estas variables, se puede posteriormente iniciar un estudio cuidadoso de los modelos de gestión implementados, que han proporcionado resultados importantes en las Ligas exitosas y, de esta forma, poder aportar en la búsqueda de un mayor equilibrio, para el desarrollo deportivo del país.

A los profesionales dedicados a la administración de los entes deportivos, como en este caso, de las Ligas, les corresponde conocer y hacer una articulación cuidadosa con las teorías administrativas, toda vez que su labor fundamental es la de liderar el recurso humano, para atender y gestionar actividades, proyectos y programas, que deben emerger tras una planeación cuidadosa, una organización detallada, un direccionamiento riguroso y un control pormenorizado, que permita la consecución de los objetivos propuestos, con el objetivo común de un mejor desarrollo deportivo del país.

Es claro que, de acuerdo con las normas correspondientes, la estructura administrativa de las ligas deportivas está estipulada, de una manera concreta, en el Decreto 1228 (1995) objeto fundamental del proyecto; sin embargo, no recayó sobre esta estructura, ya normalizada, para el caso de Colombia, sino sobre el hecho mismo de la organización administrativa lograda en estos organismos; en otras palabras, la articulación que los individuos deben lograr, de manera correcta, en los equipos de trabajo, para que se forjen propósitos y objetivos de desarrollo en las instituciones que dirigen, con metas de productividad y desarrollo, buscando, como lo dijo Gómez (1998), que las decisiones se tomen y las responsabilidades se establezcan, tan cerca de los usuarios como sea posible y que la distribución de responsabilidades esté acompañada de un "reparto congruente de poderes".

\section{MATERIALES Y MÉTODOS}

Se realizó el estudio en 12 ligas de Bogotá, de un total de 48, escogidas en un muestreo por conveniencia, por la facilidad que presentaron los directivos para el estudio y de manera no probabilística, mediante un diseño metodológico mixto (Rodríguez et al. 1996) al presentar, por una parte, una labor cuantitativa, apoyada en los procesos estadísticos, para registrar, de manera exacta, el número de datos en estudio y enumerar sus diferentes respuestas y, por otra, una labor cualitativa, desarrollada desde una perspectiva analítica, para encontrar las relaciones existentes entre la organización administrativa, el desarrollo organizacional y la productividad deportiva de las Ligas Bogotanas de Deporte.

La búsqueda sistemática, se desarrolló con los criterios propuestos por Tamayo \& Tamayo (2003), donde se compiló 
la información, a través de las preguntas de las encuestas, aplicadas a directivos de estos organismos, en sus propias oficinas; fueron recopiladas, posteriormente, en un instrumento único (Cuadro 1), relacionadas con la organización administrativa con que se dirige la institución, los logros conseguidos y el desarrollo organizacional alcanzado; después, se analizaron los resultados, para las conclusiones correspondientes.
El estudio es de alcance correlacional, donde se quisieron identificar las características del desarrollo administrativo que las Ligas deportivas han logrado, para relacionarlas con la productividad deportiva conseguida, realizando, así, un contraste de los logros obtenidos en el quehacer deportivo frente a la organización adelantada y llegar a las conclusiones necesarias, luego de reconocer cada uno de los hallazgos. A partir de las respuestas, se elaboró una estadística

Cuadro 1. Ficha de recolección de los datos de las encuestas.

\begin{tabular}{|c|c|}
\hline NOMBRE DE LA LIGA & AÑO DE CONFORMACIÓN_ \\
\hline ESTRUCTURA ADMINISTRATIVA & Formación académica de directivos \\
\hline Presidente & Logros deportivos de la administración \\
\hline Vicepresidente & Actual \\
\hline Secretario & Reuniones de asamblea general \\
\hline Tesorero & Productividad deportiva alcanzada \\
\hline Vocal & Claridad en metas y objetivos \\
\hline Revisor fiscal & Logros deportivos deficientes \\
\hline Comisión disciplinaria & Enfoque Administrativo \\
\hline Comisión técnica & Organización eficiente \\
\hline Comisión de juzgamiento & Recursos del estado \\
\hline ESTRUCTURA INTERNA & DESARROLLO ORGANIZACIONAL \\
\hline Número de Clubes inscritos & Ambiente laboral \\
\hline Número de deportistas Activos & Comunicación interna \\
\hline Escenarios deportivos adecuados & Remuneración \\
\hline Sí__ No ___ & Ambiente de confianza \\
\hline Equipo técnico & Posibilidades de desarrollo profesional \\
\hline Equipo científico & Empoderamiento \\
\hline Recursos para el Deportista & \\
\hline Capacitación a Técnicos & \\
\hline Capacitación a Directivos & \\
\hline Desarrollo profesional & \\
\hline
\end{tabular}

inferencial, usando la teoría de probabilidades, para generar las características de una muestra representativa, es decir, utilizando estadísticas muestrales fundamentales, para obtener y presentar en el estudio conclusiones generales.

\section{RESULTADOS Y DISCUSIÓN}

Las ligas deportivas en Bogotá, se regulan por estatutos similares; un ejemplo de ellas, la Liga Bogotana de Taekwondo. "La liga Bogotana de Taekwondo es un organismo deportivo de derecho privado sin ánimo de lucro, dotada de Perso- nería Jurídica, mediante Resolución N. 685 del 31-07-85 y Reconocimiento Deportivo No. 2243 del 6 de noviembre del 2015, que cumple funciones de interés público y social por delegación de la Federación Colombiana de Taekwondo". Este organismo de deporte asociado tiene como sede y domicilio el Distrito Capital de Bogotá, para el desarrollo de sus actividades.

Su objeto es "fomentar, patrocinar, dirigir, planear y organizar la práctica del deporte del Taekwondo y sus modalidades, dentro del ámbito territorial del Distrito Capital de Bogotá 
e impulsar programas de interés público y social en acatamiento a la ley 181 y al Decreto 1228 (1995) y demás disposiciones que rigen las actividades deportivas de la práctica de la disciplina deportiva del Taekwondo".

La liga está conformada, en la actualidad, por clubes deportivos, como lo expresa el capítulo III, en los artículos 9 y 10 de los estatutos, donde, entre sus disposiciones, se encuentra que cada club deberá obtener reconocimiento deportivo expedido por el I.D.R.D. para iniciar su proceso de afiliación a la liga"; sin embargo, a pesar de la normativa clara y general de los decretos y entendiendo la alta responsabilidad que tienen las Ligas en el camino del deportista, no se advierte un desarrollo armónico y equilibrado entre ellas; por el contrario, se observan grandes diferencias, en relación con la organización administrativa, el desarrollo organizacional y la productividad deportiva, de tal manera, que unas logran resultados deportivos importantes y albergan muchos deportistas en sus filas, mientras que otras, apenas si sobreviven en una evolución básica. En el aspecto financiero, también existe un desequilibrio preocupante; algunos de estos entes deportivos deben acudir a un sinnúmero de actividades, para recolectar los dineros, que permitan su sostenibilidad y desarrollo.

El estudio mostró que la estructura Administrativa de las Ligas deportivas bogotanas está acorde con lo estipulado por el Decreto 1228 (1995), de tal forma, que el 72\% del cuerpo administrativo, lo compone un presidente, un secretario y un tesorero. En las restantes, además, hay un vicepresidente y un vocal, ya que en este decreto está estipulado que el órgano de administración puede ser de tres o de cinco integrantes, incluyendo, además, la ubicación de los órganos de disciplina y de control, como corresponde.
La estructura organizativa normalizada para las ligas, les parece suficiente, en número y función, al $62 \%$ de los directivos, que respondieron las encuestas, considerando, además, que de acuerdo con esta disposición, las Ligas han obtenido logros importantes en el deporte de la ciudad; el 38\% de los encuestados está de acuerdo en que la estructura es buena, pero falta dinámica, ya que muchos de los funcionarios no desarrollan una labor administrativa concreta, es más una labor representativa, por lo tanto, no desarrollan tareas concisas, continuas y claras.

Con relación a la aplicación de esta estructura normativa, aparecen Ligas con una organización bien establecida, un organigrama amplio y reconocido por la mayoría de sus integrantes, mientras que existen otras ligas, en la misma ciudad, donde los presidentes hacen simultáneamente de entrenadores y los entrenadores son al tiempo coordinadores o tesoreros; aparecen en el estudio entes deportivos, cuya organización administrativa no es conocida por la mayoría de sus usuarios, quienes tampoco identifican su enfoque organizacional. Un porcentaje importante (30\% de los encuestados), considera que no ha habido una gestión eficiente en los últimos periodos administrativos de las Ligas Bogotanas.

Ya lo expresó Cajigal (1991), que la dirección de los deportes no se encuentra en manos de los profesionales del deporte. Esta investigación ratifica esa aseveración (Figura 1), al reconocer que el 58\% de las ligas en Bogotá están siendo dirigidas por profesionales de otras áreas del conocimiento, no por licenciados en Educación física, ni por profesionales de las ciencias del deporte y tampoco por administradores deportivos.

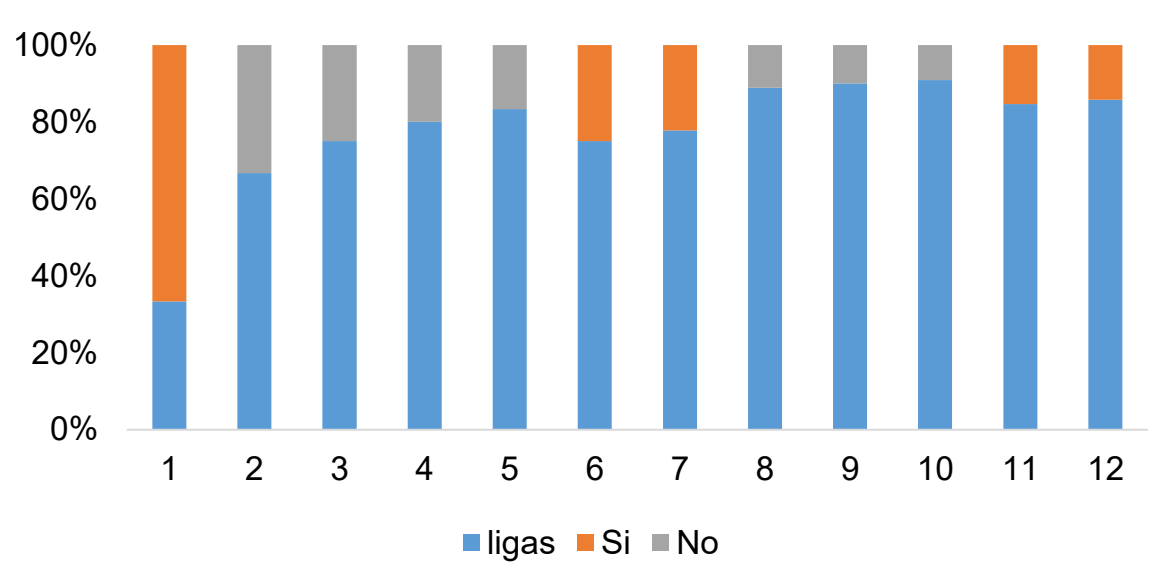

Figura 1. Ligas deportivas de Bogotá, D.C. dirigidas por personas, con título profesional en deporte. 
El estudio muestra que, en estos cargos, aparecen personas con otras profesiones, ni siquiera relacionadas con el deporte, seguramente, realizando una labor de compromiso y entrega de buenos resultados, pero con las falencias correspondientes, al no haber sido preparados académica ni profesionalmente; entonces, según Zapata Montoya et al. (2010), en una investigación de las ligas deportivas de Antioquia, "En el país cualquier persona puede postularse para un cargo de directivo en una Liga deportiva, porque no se exige en Colombia un título profesional, experiencia administrativa, conocimiento del deporte o manejo de habilidades gerenciales en general, para integrar un equipo directivo de una liga".

Por otra parte, el estado otorga unos recursos económicos básicos para el desarrollo fundamental de las Ligas deportivas y según el $100 \%$ de los directivos encuestados, estos no son suficientes, para lograr los resultados correspondientes; los directivos deben adelantar continuamente procesos de gestión económica, para posibilitar los resultados que se requieren y, aunque las ligas son entes particulares y, por lo tanto, les corresponde realizar esa gestión, un alto porcentaje $(40 \%)$ de los encuestados, aseveran que el Estado podría dar mayor apoyo, para así asegurar un mejor desarrollo para el deporte del país y para que no sea necesario implementar estrategias, que son molestas para los deportistas, como los cobros de mensualidades y matrículas, entre otras (Figura 2).

Las ligas que están iniciando su desarrollo son las que muestran mayores inconvenientes en este aspecto, adoleciendo, además, de escenarios deportivos, no tienen un número importante de deportistas que den apoyo a la gestión en general y, por lo tanto, con mucha dificultad, permanecen en el ámbito del deporte, aunque no logran consolidar su misión y sus propósitos. El mercadeo deportivo podría representar una solución importante para estas circunstancias, pero no hay facilidades en su implementación; las empresas aún no están al tanto de las bondades de la cultura del patrocinio, anulando, en muchos casos, esta estrategia de desarrollo económico.

La infraestructura de las ligas requiere de mayores y más adecuadas áreas específicas para cada deporte, para así poder hacer una mayor convocatoria a la ciudadanía, posibilitando mejores condiciones para el entrenamiento de los atletas y así tener en un futuro, más representantes deportivos de la ciudad y el país. Es necesario, en este punto, hacer hincapié, en la importancia del apoyo técnico (entrenadores, preparadores físicos, metodólogos) y el científico (médicos, nutricionistas, fisioterapeutas), que brinda el gobierno distrital, sin el cual, las Ligas deportivas no podrían tener el desarrollo que han logrado.

Tal vez por lo anterior, en el actual período administrativo, el $18 \%$ de las Ligas deportivas muestran un importante desarrollo (Figura 3), logrando un crecimiento continuo en el número de inscritos, consolidándose en la ciudad y alcanzando una excelente productividad deportiva, al ganar entre el 65 y $75 \%$ de los torneos y las copas de importancia, en el ámbito de su deporte y, ante todo, aportando deportistas a las justas olímpicas. Estos organismos presentan una organización deportiva bien implementada, donde, además de la gestión de sus directivos, tienen a su favor la labor de profesionales en administración, contabilidad y mercadeo deportivo, asegurando procesos de consolidación y de crecimiento integral, como lo sugiere Gutiérrez (2007), para el caso de administración deportiva.

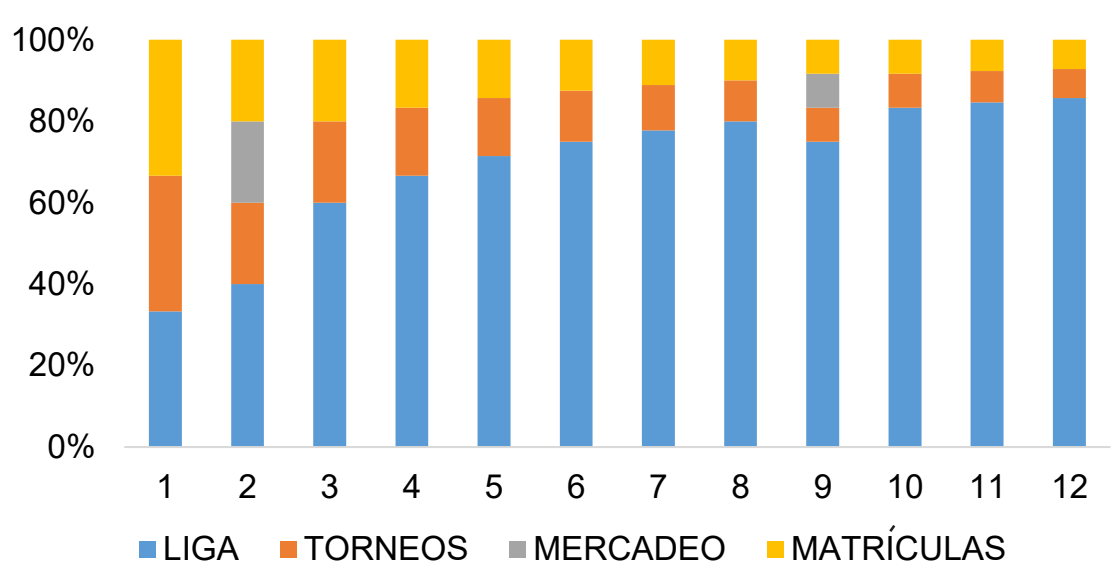

Figura 2: Recursos que deben implementar las ligas bogotanas para su supervivencia. 


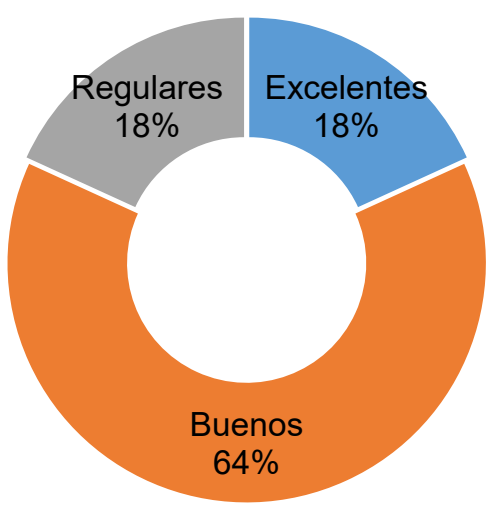

Figura 3: Resultados deportivos y desarrollo de las ligas bogotanas encuestadas en este estudio.

Un $64 \%$ de las Ligas bogotanas muestran un regular desarrollo y están en el camino de consolidarse en la ciudad, participan, de manera continua, en torneos importantes, ganando algunos de ellos; también logran aportar algunos deportistas a las olimpiadas; su organización deportiva está soportada en colaboradores que, en su gran mayoría, no son profesionales, pero han adelantado cursos técnicos, que les permite dar aportes importantes a sus entes correspondientes. El $18 \%$ restante de las Ligas deportivas, no ha logrado buen desarrollo en el último periodo administrativo. Los resultados en cuanto a torneos han sido mínimos y la consolidación en la ciudad aún es una meta por cumplir.

Con relación a la organización deportiva, se encontró que el $82 \%$ de los directivos del último periodo administrativo, dicen no conocer los logros y los resultados de la anterior administración, lo que hace pensar que no hay una planeación administrativa diseñada, para permitir un desarrollo continuo de las actividades. La planeación que se logra estructurar con base en estudios de diagnóstico y antecedentes es la que permite, con mayor seguridad, el desarrollo de las organizaciones, en general (Robbins, 1994) (Figura 4).

Un tema fundamental dentro del trabajo presente es el desarrollo organizacional que han alcanzado las Ligas en estudio y es, dentro de los elementos analizados, el que mejores resultados presenta. El $85 \%$ de las encuestas diligenciadas coincide en mostrar que, en estos entes deportivos, hay un clima laboral de gran progreso; la labor se desempeña con agrado, toda vez que se percibe respeto, confianza y camaradería en el equipo de trabajo; la remuneración, en un $70,5 \%$, es calificada como buena; las directivas facilitan a todos los empleados posibilidades de desarrollo profesional, a través de cursos de actualización y de capacitación permanente; sin embargo, un porcentaje pequeño (30\%) señala que hace falta una mejor infraestructura en oficinas y, en lo referente a una mejor tecnología, para tener más comodidades, para las labores generales. En el desarrollo organizacional, como sistema, se insiste en que hay que tomar como centro de desarrollo al recurso humano y su relación con el ambiente laboral, el clima organizacional, la cultura, la productividad, el liderazgo y el desarrollo profesional (Robbins \& Judge, 2016).

El desarrollo organizacional está en pleno ascenso en las Ligas deportivas de Bogotá. En este aspecto, los estudios manifiestan un positivo resultado, en el $82 \%$ de ellas y la productividad muestra una relación directa con ese buen desarrollo, con altos estados de motivación, relacionados con ambiente laboral, salarios e incentivos, los empleados y directivos de estas organizaciones han logrado buenos índices de productividad deportiva y administrativa, pero la preocupación sigue siendo por aquellas Ligas que no presentan una organización deportiva fuerte, que no tienen un desarrollo organizacional estable y que, por lo tanto, presentan un deficiente desarrollo deportivo, lo que incide, junto con otros elementos, en el desequilibrio existente entre unos y otros deportes en la capital.

En cuanto a la organización administrativa, no se puede mencionar un único enfoque administrativo en las ligas, ya que hay muchas diferencias marcadas entre ellas; sin embargo, el enfoque de la administración clásica es el más relacionado, toda vez que la organización de estos entes es guiada por un ciclo administrativo estructurado, de allí el énfasis en la estructura y la funcionalidad. En este sentido, el enfoque de la corriente clásica es el más aplicado en las Ligas deportivas, su organización parte de la dirección y va hacia la base, hacia los diferentes componentes, con la claridad de un jefe principal, que forma una estructura cuidadosa con sus subalternos, de acuerdo con las diferentes funciones que la organización requiere (Fayol, 1987). 


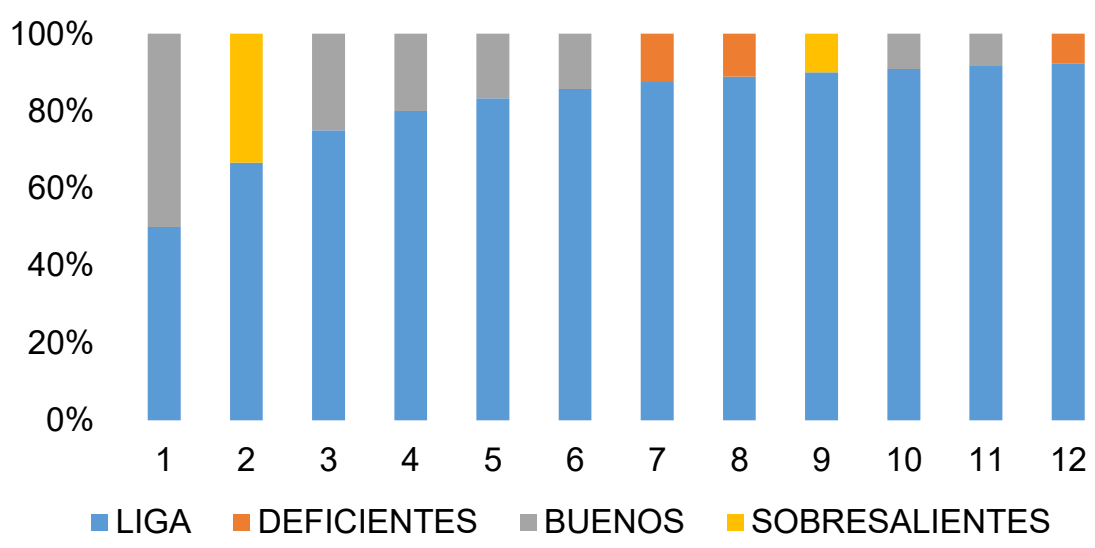

Figura 4: Logros deportivos alcanzados por las ligas bogotanas evaluadas en este estudio.

Se aprecia, que se aplican en las ligas algunos enfoques modernos de la administración, como es el Bench Marking, en el que las ligas realizan procesos de reconocimiento y de comparación con otras ligas y otras organizaciones deportivas de buen desarrollo administrativo, para conocer procedimientos exitosos y aplicarlos a sus propias organizaciones, para lograr resultados importantes, aprovechando, además, el apoyo en recursos de infraestructura, brindado por el Estado. Seguramente, sin los espacios que presta el gobierno distrital a las ligas y sin el recurso humano de apoyo a los procesos, sería imposible el desarrollo óptimo de sus actividades.

La importancia de las ligas deportivas en el país es alta, ya que en ellas, se anidan los prospectos que, más adelante, pueden representar al país en las justas departamentales, nacionales, internacionales, mundiales y olímpicas, como la dictamina la ley 181 de 1995; por lo tanto, es de suma importancia que el Estado supervise, con mayor cuidado, la productividad deportiva lograda por estas entidades, así como su organización administrativa, dada la importancia de sus aciertos, en el panorama general del deporte Colombiano, dentro de un enfoque de equidad social, que apoye la resolución de las desigualdades sociales; sin embargo, se observa, de acuerdo con los resultados del estudio, que no hay un desarrollo administrativo homogéneo en las diferentes ligas deportivas, ni tampoco una productividad deportiva similar, existe un desequilibrio inquietante entre unas y otras.

Se pueden señalar, así, algunas conclusiones del estudio. La productividad general de las ligas es diversa; se encontraron Ligas con alta productividad, mediana y deficiente productividad; este rendimiento presenta una relación directa con la organización administrativa, que logran conformar los directivos y sus empleados y, por supuesto, con el desarrollo organizacional alcanzado por estos equipos de trabajo, como ya lo había mostrado la investigación de Gutiérrez (2007), quien mostró cómo la buena organización administrativa influye directamente en los logros de los entes deportivos en Antioquia y como también lo señaló Taylor (1971), en sus fundamentos: "Es importante cultivar un buen ambiente laboral en la organización entre la gerencia y los trabajadores para mejorar también así la productividad" y Chiavenato (2004) en su tratado acerca de la teoría general de la administración.

Las Ligas de alta productividad son también las ligas que tienen un avanzado desarrollo organizacional; los empleados, se encuentran empoderados con sus cargos y logran así una labor de alto compromiso, corroborando que el rendimiento de los individuos, su satisfacción y su productividad es determinado, de alguna manera, por el ambiente laboral y la organización que impera en la institución (Blau \& Scott, 2002).

El desarrollo organizacional alcanzado en estas Ligas deportivas es de muy buenas características; existe en ellas, en general, un gran ambiente laboral, lo que, junto con la buena gestión administrativa, presenta como resultante una valiosa productividad deportiva. Así lo enunció Koontz et al. (2012), la productividad es relacionada fundamentalmente con el control, la supervisión y la evaluación constante de los procesos administrativos; las organizaciones son conformadas por grupos de personas que, en cuanto más tengan objetivos comunes, más dan de sí mismas, para conseguir beneficios mutuos y de la institución (Robbins \& Judge, 2016).

El panorama de variabilidad productiva de estos organismos deportivos es preocupante y, además, no hay un desarrollo equilibrado de organización entre ellos; hay muchos deportistas con grandes talentos deportivos en ligas que no presentan progreso y, así, el talento se pierde. Le corresponde al Estado observar estas circunstancias o estará el país 
deportivo a expensas de estar representado por los mismos deportes siempre y por los mismos deportistas; el aporte de la Nación a estos entes privados y la normativa existente, le permitir posibilidades de control y de evaluación, que este no aplica en favor de los procesos de producción, donde los componentes fundamentales son la efectividad y la eficiencia en el desempeño individual y organizacional. De acuerdo con Drucker (1996), en el área específica de la Administración deportiva y, en este caso concreto, de las ligas, la productividad se mide por los resultados logrados con el presupuesto que tiene para el periodo correspondiente $\mathrm{y}$, en este aspecto, las ligas deportivas presentan grandes diferencias y varios contrastes.

La administración de las ligas, de acuerdo con la Carta Internacional de la Educación Física (UNESCO, (2015), requiere de profesionales del área en su direccionamiento para su óptimo funcionamiento, profesionales que vayan más allá de simplemente ejecutar los presupuestos y tratar de conseguir los objetivos deportivos básicos; las ligas pueden impulsar programas que fortalezcan, socialmente, a las poblaciones, donde se desenvuelven y desarrollen realmente un deporte social formativo, teniendo en cuenta que los programas de estos organismos deportivos hacen parte del plan nacional del deporte y que, por lo tanto, se espera de ellos un aporte mayor al que están dando (Ley 181 de 1995).

De acuerdo con lo anterior, se deja a consideración, la importancia de diseñar sistemas de medición, que permitan supervisar y evaluar los logros y la productividad deportiva de las Ligas, de su entorno y su organización administrativa, para poder estar al tanto del desarrollo deportivo de cada uno de estos organismos y poder así aportar asesoría y control estatal continuo por parte del gobierno Nacional, para corregir los desequilibrios inquietantes, encontrados entre unas y otras Ligas, entre unos y otros deportes.

Conflictos de intereses: El manuscrito fue preparado y revisado por el autor, quien declara que no existe conflicto de intereses que ponga en riesgo la validez de los resultados presentados.

\section{REFERENCIAS}

1. BLAU, P.; SCOTT, W.R. 2002. Comportamiento y desarrollo organizacional. San Francisco: Chandler Publishing Co.

2. CAJIGAL, J. 1991. Oh Deporte. Anatomía de un gigante. Ed Miñón, Madrid, España. 234p.

3. DECRETO 1228 DE 1995. 1995.Por el cual se revisa la legislación deportiva vigente y la estructura de los organismos del sector asociado con objeto de ade- cuarlas al contenido de la Ley 181 de 1995. Diario Oficial No 41.933. 11p.

4. CHIAVENATO, I. 2004. Introducción a la teoría general de la Administración. Ed McGraw Hill. México, D.F. 298p.

5. CONSTITUCIÓN POLÍTICA DE COLOMBIA 1991. 2016. Actualizada con los Actos Legislativos a 2016. Ed. Corte Constitucional Consejo Superior de la Judicatura. Centro de Documentación Judicial -CENDOJ. Biblioteca Enrique Low Murtra- BELM.

6. DRUCKER, P. 1996. La Administración en una época de grandes Cambios. Ed, Sudamericana. Argentina. 248p.

7. FAYOL, H. 1987. Administración industrial y general. $1^{\mathrm{a}}$ ed. Buenos Aires, Ed. Ateneo. 46p.

8. GÓMEZ, H. 1998. Educación: la Agenda del Siglo XXI: hacia un desarrollo humano. Programa Naciones Unidas. Ed, TM. Bogotá. 366p.

9. GUTIÉRREZ BEANCUR, J.F. 2007. Fundamentos de Administración Deportiva. Universidad de Antioquia. Instituto Universitario de Educación Física. Medellín, Colombia. 107p.

10. KOONTZ, H.; WEIHRICH, H.; CANNICE, M. 2012. Administración, una perspectiva global y empresarial. 14. ed. México, Ed. Mc Graw Hill. 706p.

11. LEY 181 de Enero 18 de 1995. 1995. Por el cual se dictan disposiciones para el fomento del deporte, la recreación, el aprovechamiento del tiempo libre y la Educación Física y se crea el Sistema Nacional del Deporte. 18 de enero de 1995. 233p.

12. MARTÍNEZ CRESPO, J. 2005. Administración y Organizaciones. Su desarrollo evolutivo y las propuestas para el nuevo siglo. Semestre Económico. 8(16):6797.

13. MESA, J.; ARBOLEDA, R.; GAVIRIA, N.; GUZMÁN, K. 2010. Estado de Desarrollo de las organizaciones deportivas colombianas. Universidad de Antioquia. 138p.

14. OMANA DEL CASTILLO, R. 2003. Del proyecto a la realidad. Ed. el Sol, Monterrey, México Revista el Norte. $6 \mathrm{p}$. 
15. RODRÍGUIEZ, G.; GIL, J.; GARCIA, E. 1996. Metodología de la Investigación Cualitativa. Ed Aljibe, Granada. España. 380p.

16. ROBBINS, S.P. 1994. Administración teoría y Práctica. $4^{\mathrm{a}}$ ed. México. Prentice-Hall Hispanoamericana. 560p.

17. ROBBINS, S.P.; JUDGE, T.A. 2016. Comportamiento organizacional. México. $15^{\mathrm{a}}$ ed. Ed Pearson. 712p.

18. TAMAYO Y TAMAYO, M. 2003. El proceso de la Investigación Científica, México, Ed Limusa Noriega eds. $175 p$.

19. TAYLOR, F.W. 1971. Principios de la Administración Científica. Ed Herrero Hermanos. México.
20. UNESCO. 2015. Carta Internacional de La Educación Física, la Actividad Física y el Deporte. Carta de las Naciones Unidas sobre la Educación Física, la Actividad Física y el Deporte. 10p. Disponible desde Internet en: http://unesdoc.unesco.org/ images/0023/002354/235409s.pdf (con acceso 08/04/2018)

21. ZAPATA MONTOYA, G.L.; ZAPATA CALDERÓN, G.A.; GÓMEZ ZAPATA. Y. 2010. Gestión y características administrativas de las ligas deportivas del departamento de Antioquia 2000-2010. Revista Politécnica. 6(11):25-37.

Recibido: Febrero 22 de 2018

Aceptado: Noviembre 12 de 2018

\section{Cómo citar:}

Ramos Acosta J. 2018. Organización administrativa y productividad de las ligas deportivas bogotanas. Rev. U.D.C.A Act. \& Div. Cient.21(2):319-327. https://doi.org/10.31910/rudca.v21.n2.2018.1081 